\title{
A NOTE ON NONLINEAR SYSTEMS WITH DIFFERENT DEGREES OF EXCITATIONS
}

\author{
NGUYEN VAN DAO \\ Institute of Mechanics, NCNST of Vietnam
}

In this note an approximate solution of a differential equation of special form which is close to the Liapunoff type is given. This solution is proportional to the small parameter $\varepsilon$.

\section{DIFFERENTIAL EQUATION AND ITS APPROXIMATE SOLUTION}

Let us consider a nonlinear system described by the differential equation

$$
\ddot{x}+\omega^{2} x=\gamma x^{2}-\beta x^{3}-\varepsilon^{2} h \dot{x}+\varepsilon^{3} f(\varphi, x, \dot{x}), \quad \varphi=\nu t
$$

where $\omega, \gamma, \beta, h, \nu$ are constants, $\varepsilon$ is a small positive parameter, $f(\varphi, x, \dot{x})$ is a periodic function relatively to $t$ and analytic to $x, \dot{x}$. We are intersted in finding the periodic solution of the equation (1.1). It is noted that when $\varepsilon=0$ the equation (1.1) is degenerated into

$$
\ddot{x}+\omega^{2} x=\gamma x^{2}-\beta x^{3}
$$

which has a trivial stable solution $x=0$. Hence, the solution of the equation (1.1) is found in the form:

$$
x=\varepsilon a \cos \left(\frac{p}{q} \varphi+\psi\right)+\varepsilon^{2} u_{1}(a, \varphi, \theta)+\varepsilon^{3} u_{2}(a, \varphi, \theta)+\ldots,
$$

where $\theta=\frac{p}{q} \varphi+\psi, \frac{p}{q} \nu \simeq \omega, p$ and $q$ are integers, $u_{i}(a, \varphi, \theta)$ are periodic functions of $\varphi, \theta$ and do not contain the first harmonics $\sin \theta, \cos \theta$. The amplitude $a$ and phase $\psi$ are determined from the equations of form

$$
\begin{aligned}
\frac{d a}{d t} & =\varepsilon A_{1}(a, \psi)+\varepsilon^{2} A_{2}(a, \psi)+\ldots \\
\frac{d \psi}{d t} & =\omega-\frac{p}{q} \nu+\varepsilon B_{1}(a, \psi)+\varepsilon^{2} B_{2}(a, \psi)+\ldots
\end{aligned}
$$

To find the unknown functions $u_{i}, A_{i}, B_{i}$ we calculate the derivatives $\dot{x}, \ddot{x}$ from (1.3) and substitute them and (1.3), (1.4) into (1.1). By comparing the coefficients of $\varepsilon^{2}$ we obtain

$$
\begin{aligned}
& \left(\nu \frac{\partial}{\partial \varphi}+\omega \frac{\partial}{\partial \theta}\right)^{2} u_{1}+\omega^{2} u_{1}=\gamma a^{2} \cos ^{2} \theta \\
& -\left[\left(\omega-\frac{p}{q} \nu\right) \frac{\partial A_{1}}{\partial \psi}-2 \alpha \omega B_{1}\right] \cos \theta+\left[\left(\omega-\frac{p}{q} \nu\right) a \frac{\partial B_{1}}{\partial \psi}+2 \omega A_{1}\right] \sin \theta
\end{aligned}
$$

Comparing the coefficients of the harmonics in (1.5) we have 


$$
A_{1}=0, \quad B_{1}=0, \quad, u_{1}=\frac{\gamma a^{2}}{2 \omega^{2}}\left(1-\frac{1}{3} \cos 2 \theta\right) .
$$

If one compares the coefficients of $\varepsilon^{3}$ in (1.1) one has

$$
\begin{aligned}
& \left(\nu \frac{\partial}{\partial \varphi}+\omega \frac{\partial}{\partial \theta}\right)^{2} u_{2}+\omega^{2} u_{2}=2 a \gamma u_{1} \cos \theta+f(\varphi, a \cos \theta,-a \omega \sin \theta)- \\
& -\left[\left(\omega-\frac{p}{q} \nu\right) \frac{\partial A_{2}}{\partial \psi}-2 a \omega B_{2}\right] \cos \theta+\left[\left(\omega-\frac{p}{q} \nu\right) a \frac{\partial B_{2}}{\partial \psi}+2 \omega A_{2}\right] \sin \theta
\end{aligned}
$$

The form of functions $A_{2}, B_{2}$ and $u_{2}$ depends on the concrete form of the function $f(\varphi, x, \dot{x})$.

\section{FORCED OSCILLATION}

Let us consider the case when the function $f(\varphi, x, \dot{x})$ is of the form

$$
f(\varphi, x, \dot{x})=E \sin \nu t
$$

and consider the resonant case $p=q=1$ :

$$
\nu \simeq \omega
$$

Comparing the coefficients of harmonics in (1.7) we have

$$
\begin{aligned}
& (\omega-\nu) \frac{\partial A_{2}}{\partial \psi}-2 a \omega B_{2}=\frac{5}{6} \frac{\gamma^{2} a^{3}}{\omega^{2}}-E \sin \psi, \\
& (\omega-\nu) a \frac{\partial B_{2}}{\partial \psi}+2 \omega A_{2}=-E \cos \psi, \\
& \left(\nu \frac{\partial}{\partial \varphi}+\omega \frac{\partial}{\partial \theta}\right)^{2} u_{2}+\omega^{2} u_{2}=-\frac{1}{6} \frac{\gamma^{2} a^{3}}{\omega^{2}} \cos 3 \theta .
\end{aligned}
$$

From the equations (2.3) one obtaines

$$
\begin{aligned}
& A_{2}=\frac{-E \cos \psi}{\nu+\omega} \\
& B_{2}=-\frac{5 \gamma^{2} a^{2}}{12 \omega^{3}}+\frac{E \sin \psi}{(\nu+\omega) a}, \\
& u_{2}=\frac{\gamma^{2} a^{3}}{48 \omega^{4}} \cos 3 \theta
\end{aligned}
$$

Hence, the solution of the equation (1.1) in the second approximation is

$$
x=\varepsilon a \cos (\nu t+\psi)+\varepsilon^{2} \frac{\gamma a^{2}}{2 \omega^{2}}\left(1-\frac{1}{3} \cos 2 \theta\right)+\varepsilon^{3} \frac{\gamma^{2} a^{3}}{48 \omega^{4}} \cos 3 \theta,
$$

where $a$ and $\psi$ are determined from the system:

$$
\begin{aligned}
\frac{d a}{d t} & =-\varepsilon^{2} \frac{E \cos \psi}{\nu+\omega}, \\
\frac{d \psi}{d t} & =\omega-\nu-\frac{5 \varepsilon^{2}}{12} \frac{\gamma^{2} a^{2}}{\omega^{3}}+\frac{\varepsilon^{2} E \sin \psi}{a(\nu+\omega)} .
\end{aligned}
$$

These equations can be easily solved. 


\section{PARAMETRIC OSCILLATION}

We suppose that $f(\varphi, x, \dot{x})$ has the form

$$
f(\varphi, x, \dot{x})=e x \cos \nu t
$$

Ind consider the resonant case $p=1, q=2$ :

$$
\nu \simeq 2 \omega
$$

The equations for $A_{2}, B_{2}$ and $u_{2}$ are

$$
\begin{aligned}
& \left(\omega-\frac{\nu}{2}\right) \frac{\partial A_{2}}{\partial \psi}-2 a \omega B_{2}=\frac{5}{6 \omega^{2}} \gamma^{2} a^{3}+\frac{1}{2} e a \cos 2 \psi, \\
& \left(\omega-\frac{\nu}{2}\right) a \frac{\partial B_{2}}{\partial \psi}+2 \omega A_{2}=-\frac{1}{2} e a \sin 2 \psi \\
& \left(\nu \frac{\partial}{\partial \varphi}+\omega \frac{\partial}{\partial \theta}\right)^{2} u_{2}+\omega^{2} u_{2}=-\frac{1}{6 \omega^{2}} \gamma^{2} a^{3} \cos 3 \theta+\frac{1}{2} e a \cos (\theta+\varphi) .
\end{aligned}
$$

Solving these equations gives:

$$
\begin{aligned}
& A_{2}=-\frac{e a}{2 \nu} \sin 2 \psi, \quad B_{2}=-\frac{5}{12 \omega^{3}} \gamma^{2} a^{2}-\frac{e}{2 \nu} \cos 2 \psi, \\
& u_{2}=\frac{-e a}{2 \nu(\nu+2 \omega)} \cos (\theta+\varphi)+\frac{\gamma^{2} a^{3}}{48 \omega^{4}} \cos 3 \theta .
\end{aligned}
$$

The solution of the equation (1.1) in the second approximation is then

$$
x=\varepsilon a \cos \left(\frac{1}{2} \nu t+\psi\right)+\varepsilon^{2} \frac{\gamma a^{2}}{2 \omega^{2}}\left(1-\frac{1}{3} \cos 2 \theta\right)-\frac{\varepsilon^{3} e a}{2 \nu(\nu+2 \omega)} \cos (\theta+\varphi)+\frac{\varepsilon^{3} \gamma^{2} a^{3}}{48 \omega^{4}} \cos 3 \theta,
$$

where $a$ and $\psi$ satisfy the equations:

$$
\begin{aligned}
\frac{d a}{d t} & =-\frac{\varepsilon^{2} e a}{2 \nu} \sin 2 \psi \\
\frac{d \psi}{d t} & =\omega-\frac{1}{2} \nu-\frac{5 \varepsilon^{2}}{12 \omega^{3}} \gamma^{2} a^{2}-\frac{\varepsilon^{2} e}{2 \nu} \cos 2 \psi
\end{aligned}
$$

\section{MộT NHẬN XÉT VỀ HỆ PHI TUYẾN} VớI CÁC BẬC Kf́CH ĐộNG KHÁC NHAU

Trong bài đã đưa ra một lời giải xấp xỉ có dạng đặc biệt cho phương trình vi phân gần với Liapunôp. Hai trường hợp của dao động cưỡng bức và dao động thông số đã được khảo sát. 